\title{
BUBBLE ELIMINATION BY SWIRL FLOW
}

\author{
Ryushi Suzuki*, Kunio Matsui** \\ Yoshihiro Mochimaru*** \\ *Stuff Dept., Omnipac Electronics \\ Tachikawa, Japan \\ **Ishikawajima-Harima Heavy Industries \\ Research Institute \\ Tokyo, Japan \\ ***Dept. of Mechanical Engineering \\ Tokyo Institute of Technology \\ Tokyo, Japan
}

ABSTRACT

The performance of a newly developed bubble elimination system is analyzed. The system consists of a circular body for a swirled flow supplied tangentially, equiped with a throttle valve downward and a valve for discharging bubbles upward.

A strong swirl superimposed on an averaged laminar flow during the initial state is found to decay exponentially with the distance from the entrance at the far downstream. As a result, for Reynolds numbers greater than a suitable value an adverse pressure gradient is produced, exerting a backward force on the bubbles to be discharged.

In addition, universal velocity distributions and pressure contours downward are shown.

KEY WORDS: bubble elimination, gas separation, bubble removal, swirl flow, swirling motion

\section{INTRODUCTION}

Gases contained in a fluid are causes of aeration, and increase apparent compressibility of the fluid, and decrease dynamic characteristics. Accordingly, various methods have been proposed to eliminate gas from fluid for an oil hydraulic system.

In this paper treated is an analysis of the flow in a newly developed bubble elimination method by swirl flow. By means of this method, the gas dissolved in the fluid is discharged out of fluid. Since bubbles appear in phenomena relating with cavitation and aeration, upto this time many theoretical and experimental researches on the dynamics of bubbles have been made from various view point.

Namely, Plesset et. al.[1] reviewed bubble dynamics and cavitation, and Harper[2] threw light on the motion of bubbles under the state of gravity.

\section{NOMENCLATURE}

a: inner radius of a cylindrical body

$\overrightarrow{\mathbf{g}}$ : acceleration vector of gravity

$p: \quad[\mathrm{Pressure}-\rho \overline{\mathrm{g}} \cdot(\mathrm{p} \circ \mathrm{s}$ i t i o n vector $)] /\left(\rho U_{0}^{2}\right)$, (dimensionless form)

$p_{z}{ }^{*}: \equiv(\partial p / \partial z)_{\mathrm{r}=0} \cdot \mathrm{e}^{2 \beta_{1}} \mathrm{Z}_{/ C_{1}{ }^{2}}$

$r$ : radius in a dimensionless cylindrical polar coordinate

$R e$ : Reynolds number $\equiv U_{0} a / v$

$u$ : $r$-compnent of velocity (dimensionless form)

$U_{0}$ : Mean velocity in the $z$-direction

$u^{*}: \quad \equiv u e^{2 \beta_{1} Z / C_{1}^{2}}$

$v: \quad \theta$-component of velocity (dimensionless form)

$v^{*}: \quad \equiv v \beta^{\beta_{1}} z / C_{1}^{2}$

$w: \quad z$-component of velocity(dimensionless form)

$w^{*}: \equiv(w-1) \mathrm{e}^{2 \beta_{1} z / C_{1}{ }^{2}}$

$z$ : axial coordinate in a dimensionless cylindrical polar coordinate

$\theta$ : amplitude coordinate in a dimensionless cylindrical polar coordinate

$v: \quad$ kinematic viscosity

$\rho:$ density of fluid

$\psi$ : axisymmetric dimensionless stream function defined by equation (10)

$\Delta:$ dimensionless axisymmetric Laplacian operator $\equiv \partial^{2} / \partial r^{2}+(1 / r)(\partial / \partial r)+\partial^{2} / \partial z^{2}$

ANALYSIS

Outline of a bubble elimination system

Figure 1 illustrates a schematic diagram of a proposed system which consists of a cylindrical body for swirl, an inlet pipe throttle valve on discharge line, and a vent valve for coalesced bubbles. Area of the cross section of the fluid inflow pipe toward the cylindrical body is smaller than that of the cylindrical vessel for swirl. The centerline of the inlet pipe is perpendicular to but not coplane with that 
610

of the cylindrical body, and the inlet pipe is disposed close to the lid, so that a fluid flow into a cylindrical body creates a swirl flow.

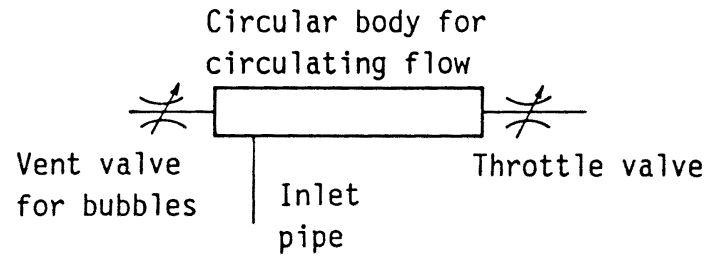

Figure 1. Schematic diagram for a bubble elimination system.

A fluid containing bubbles therein is introduced through the inlet pipe into the cylindrical body. Bubbles have tendency to halt in the vicinity of the upstream end of the swirl flow and to grow into coherent gas, so that the concentrated gas is to be discharged if a gas vent valve is provided with suitable opening. After a part of bubbles are separated, the fluid proceeds downstream through a throttle valve.

\section{Analysis of flow field}

In this section, a flow field in the cylindrical body is analyzed, using a cylindrical polar coordinate $(r, \theta, z)$ such that the $z$-axis is coincident with the centerline of the cylindrical body, the origin being located at the upstream end and the positive direciton of the $z$-axis being downward. Here, $r$ and $z$ are assumed to be made dimensionless with respect to the inner radius $a$ of the cylindrical body. Fluid flow is assumed to be incompressible, steady, and laminar, and velocity is made dimensionless with respect to the average velocity $U_{0}$ in the cylindrical seciton. All the more, flow is assumed to be axisymmetric, which is considered good almost everywhere except at the region very near the inlet port and throttle valve. Then, equations of motion in the $r, \theta, z-$ directions can be expressed as

$$
\begin{aligned}
& u \frac{\partial u}{\partial r}+w \frac{\partial u}{\partial z}-\frac{v^{2}}{r}=-\frac{\partial p}{\partial r}+\frac{1}{R e}\left(\Delta u-\frac{u}{r^{2}}\right)---(1) \\
& u \frac{\partial v}{\partial r}+w \frac{\partial v}{\partial z}+\frac{u v}{r}=\frac{1}{R e}\left(\Delta v-\frac{v}{r^{2}}\right)-----(2) \\
& u \frac{\partial w}{\partial r}+w \frac{\partial w}{\partial z}=-\frac{\partial p}{\partial z}+\frac{1}{R e} \Delta w----- \text { (3) }
\end{aligned}
$$

respectively.

The equation of continuity is

$$
\frac{\partial u}{\partial r}+\frac{u}{r}+\frac{\partial w}{\partial z}=0
$$

With the aid of Eq. (4), $p$ can be eliminated from Eqs. (1) and (3) to give

$$
\left(u \frac{\partial}{\partial r}+w \frac{\partial}{\partial z}-\frac{u}{r}\right)\left(\frac{\partial u}{\partial z}-\frac{\partial w}{\partial r}\right)-\frac{1}{r} \frac{\partial}{\partial z} v^{2}
$$

$$
=\frac{1}{R e}\left\{\frac{\partial}{\partial z}\left(\Delta u-\frac{u}{r^{2}}\right)-\frac{\partial}{\partial r} \Delta w\right\}-----
$$

In the following, a decaying process of swirl flow generated at the inlet part is examined. Since the radial component of velocity decreases in its magnitude due to viscosity as fluid passes downstream, the inertial term of Eq.(2) is approximated as $(\partial v) /(\partial z)$ just as in the Osceen approximation. Then Eq.(2) becomes

$$
\frac{\partial v}{\partial z}=\frac{1}{R e}\left(\Delta v-\frac{v}{r^{2}}\right)
$$

Considering no slip condition at $r=1$ and finite of $v$, the solution of Eq.(6) can be expresed as

$$
\begin{aligned}
& v=\sum_{n} C_{n} J_{1}\left(\mathrm{a}_{n} r\right) e^{-\beta_{n}^{2}}-\cdots \\
& a_{n}^{2}=\beta_{n}^{2}+\beta_{n} \operatorname{Re} \quad\left(\beta_{n}>0\right)-\ldots-\cdots
\end{aligned}
$$

where $a_{n}$ is the nth positive zero of the Bessel function of the first kind of order 1.

Applying similar approximation to the first term of the left-hand side of Eq.(5) gives

$$
\frac{\partial}{\partial z}\left(\frac{\partial u}{\partial z}-\frac{\partial w}{\partial r}\right)-\frac{1}{r} \frac{\partial}{\partial z} v^{2}=\frac{1}{R e}\left\{\frac{\partial}{\partial z}\left(\Delta u-\frac{u}{r^{2}}\right)-\frac{\partial}{\partial r} \Delta w\right\} \text { (9) }
$$

To estimate the intensity of velocity components $u$ and $w$, it is assumed that deviation of velocity component $w$ from a uniform velocity profile is small. Then, in the case of $|r|<1$, from Eq. (4) we get

$$
\begin{aligned}
& w=1+\frac{1}{r} \frac{\partial \Psi}{\partial r} \\
& u=-\frac{1}{r} \frac{\partial \Psi}{\partial z}
\end{aligned}
$$

For clarifying the flow nature throughout the field, as for velocity $v$ of Eq. (7) only one term corresponding to $n=1$ which indicates the slowest decay is adopted. Then the stream function takes the following form:

$$
\psi=\phi(r) e^{-2 \beta 1^{2}}
$$

From Eqs. (7) (11), the differential equation for $\phi(r)$ is expressed as follows:

$$
\begin{aligned}
& \left(\frac{d^{3}}{d r^{3}}+\frac{1}{r} \frac{d^{2}}{d r^{2}}-\frac{1}{r^{2}} \frac{d}{d r}+4 \beta_{1}^{2} \frac{d}{d r}\right)\left(\frac{1}{r} \frac{1}{d r} \phi\right) \\
& +4 \beta_{1}^{2}\left(\frac{d^{2}}{d r^{2}}+\frac{1}{r} \frac{d}{d r}+4 \beta_{1}^{2}-\frac{1}{r^{2}}\right)\left(\frac{\Phi}{r}\right)+\operatorname{Re}\left(2 \beta_{1}\right)^{3} \frac{\Phi}{r} \\
& +2 \beta_{1} \operatorname{Re} \frac{d}{d r}\left(\frac{1}{r} \frac{d \Phi}{d r}\right)=-2 \beta_{1} \operatorname{Re} C_{1}^{2} \frac{1}{r} J_{1}^{2}\left(\alpha_{1} r\right)--
\end{aligned}
$$

The boundary conditions for $\phi$ can be expressed as 
$\phi(0)=\phi^{\prime}(0)=\phi(1)=\phi^{\prime}(1)=0$

The $z$-component of gradient of $p$ along the centerline becomes

$$
\left(\frac{\partial p}{\partial z}\right)_{r=0} \approx \frac{1}{R e} \Delta w-\frac{\partial w}{\partial z}
$$

$$
\left.=\mid \frac{2}{3 R e} \phi^{\prime \prime \prime}(0)+\left(\frac{4 \beta_{1}^{2}}{R e}+2 \beta_{1}\right) \phi^{\prime \prime}(0)\right] e^{-2 \beta_{1} z}
$$
becomes

The $r$-component of gradient of $p$

$$
\begin{aligned}
\frac{\partial p}{\partial r} & \approx \frac{1}{R e}\left(\Delta u-\frac{u}{r^{2}}\right)+\frac{v^{2}}{r}-\frac{\partial u}{\partial z} \\
& =\left[\frac{2 \beta}{R e}\left\{\frac{1}{r} \phi^{\prime \prime}(r)-\frac{1}{r^{2}} \phi^{\prime}(r)+\frac{4 \beta_{1}^{2}}{r} \phi(r)\right\}\right. \\
& \left.+4 \beta_{1}^{2} \frac{1}{r} \phi(r)+\frac{1}{r} C_{1}^{2} J_{1}^{2}\left(a_{1} r\right)\right] e^{-2 \beta_{1}^{z}}+O\left(e^{-4 \beta_{1}^{z}}\right)-
\end{aligned}
$$

\section{Result of numerical analysis}

Figure 2 shows normalized velocity distributions $u^{*}(r ; R e), v^{*}(r ; R e), w^{*}(r ; R e)$ for a Reynolds number of 500 . $p_{z}^{*}$.

Figure 3 shows relation between $R e$ and

Countour of $\{p(r, z)-p(0, \infty)\} / C_{1}^{;}$at $R_{e}=500$ is shown in Figure 4.

\section{Experimental setup}

Figure 5 shows an outline of an experimental setup. Measurement of pressure distribution along the centerline of the cylindrical body was carried out by means of a Pitot tube. The fluid used was hydraulic oil of viscosity VG46 and during the test its temperature was kept within $40 \pm 2^{\circ} \mathrm{C}$. One of the data obtained is shown in Figure 6.

\section{Discussion}

Comparison between experimental and analytical pressure distributions along the centerline is shown in Figure 6 , although the assumption made in the analysis is not necessarily satisfied in the vicinity of $z=0$.

As far as the assumptions made in the analysis are concerned, the value $C_{1}$ becomes the order of the ratio of tangential inflow velocity to the cross

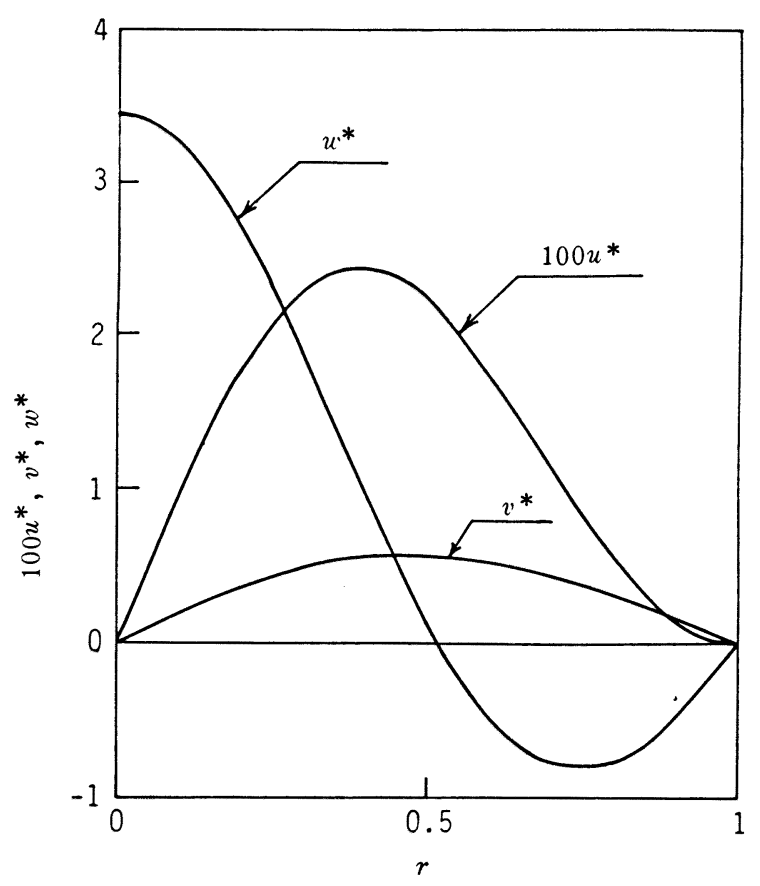

Figure 2. Normalized velocity distributions $u^{*}, v^{*}$ and $w^{*}$ as a function of $r$ at $\operatorname{Re}=500$.

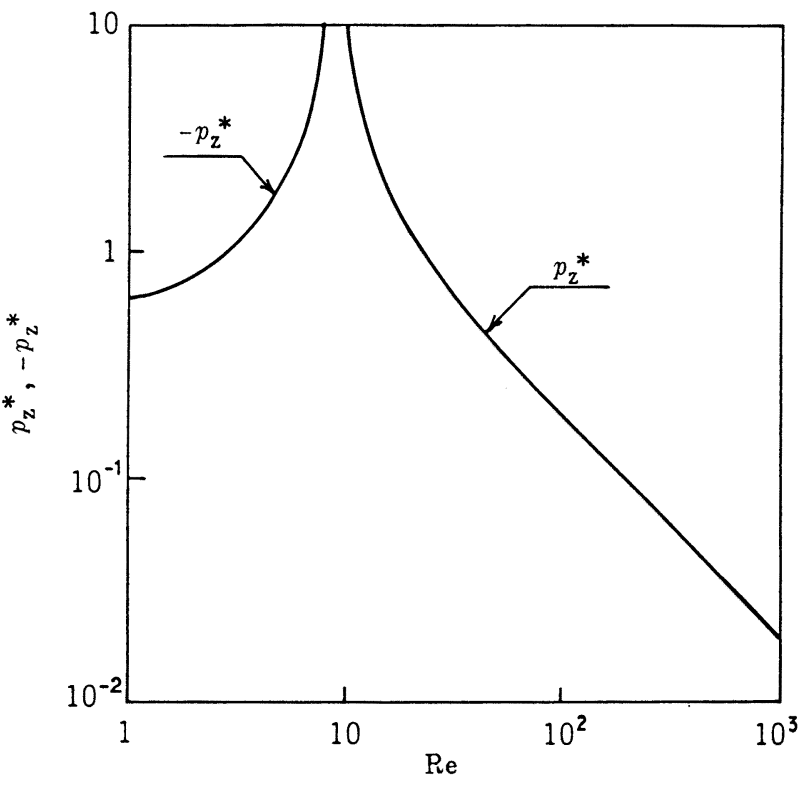

Figure 3. Normalized pressure gradient $p^{*}{ }_{2}$ as a function of Re.

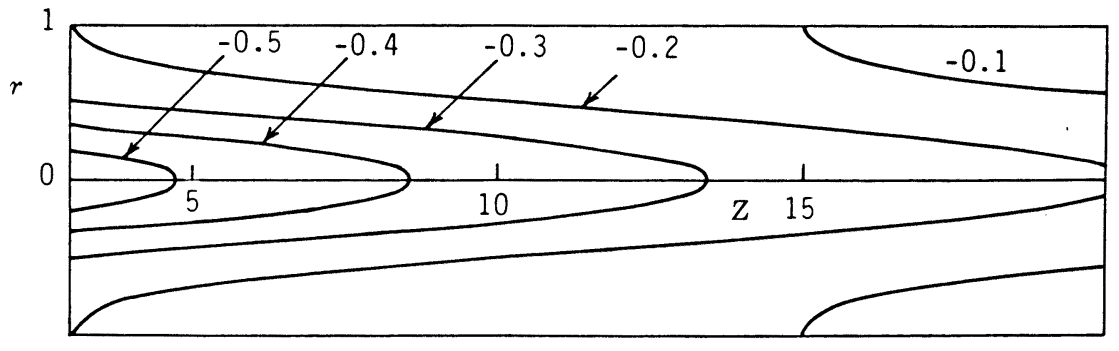

Figure 4. Contour of $[p(r, z)-p(0, \infty)] / C_{1}^{2}$ at $\operatorname{Re}=500$. 


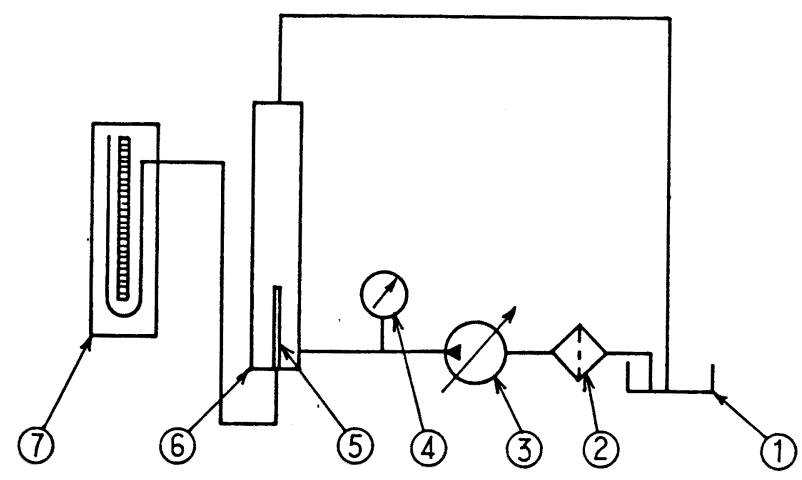

1. Reservoir

3. Pump

5. Pressure sencor

7. Manometer

Figure 5. Schematic diagram of experimental setup

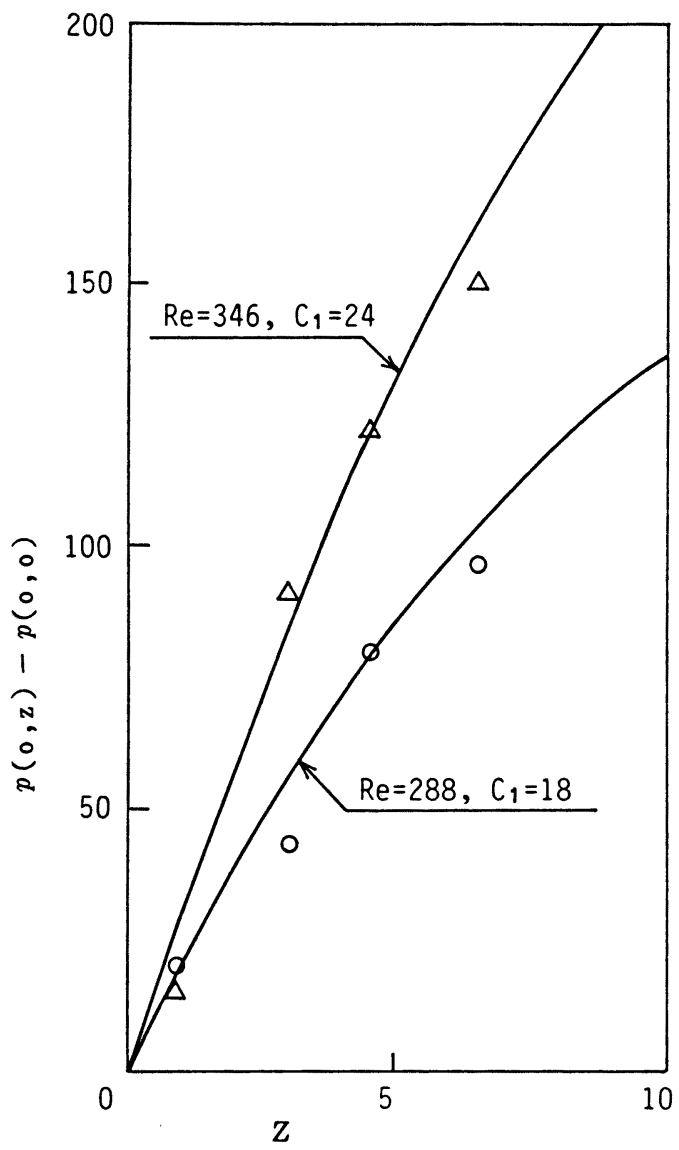

Figure 6. Distribution of $p$ along the center line.

- : analytical;

$\triangle$ : experimental $(\mathrm{Re}=346)$;

$O$ : experimental $(\mathrm{Re}=288)$

sectional average velocity in the cylindrical body and consequently depends on the experimental setup.

Since the force acted on the unit volume of bubbles is given as -(static pressure gradient) if fluid is assumed to transfer with bubbles at the same velocity, it can be recognized from Figure 3 that in the region of $P_{z}^{*}>0$ backward force is acted upon bubbles located within the cylindrical body.

\section{CONCLUSIONS}

If swirling motion is superimposed on the axial flow through a cylindrical body at a point so that $\left|C_{1}\right| \geqq 1$, then the backward force is applied upon bubbles due to the reverse pressure gradient as long as the Reynolds number $R e$ is greater than a suitably determined critical value.

Accordingly, bubbles stay or move to the backward direction and can be removed from a vent valve equipped at the upward lid of cylindrical body.

\section{REFERENCES}

[1] M.S.Plesset, A.Prosperetti: Ann. Rev. Fluid Mech., Vol.9, 145/185 (1977)

[2] J.F.Happer: Adv. in Appl. Mech., Vol.12, 59/129 (1972) 